\title{
Design of Fuzzy Logic Control System for Segway Type Mobile Robots
}

\section{Sangfeel Kwak and Byung-Jae Choi}

Department of Electronics Engineering, Daegu University, Gyeongbuk, Korea

\section{]jfis}

\begin{abstract}
Studies on the control of inverted pendulum type systems have been widely reported. This is because this type of system is a typical complex nonlinear system and may be a good model to verify the performance of a proposed control system. In this paper, we propose the design of two fuzzy logic control systems for the control of a Segway mobile robot which is an inverted pendulum type system. We first introduce a dynamic model of the Segway mobile robot and then analyze the system. We then propose the design of the fuzzy logic control system, which shows good performance for the control of any nonlinear system. In this paper, we here design two fuzzy logic control systems for the position and balance control of the Segway mobile robot. We demonstrate their usefulness through simulation examples. We also note the possibility of simplifying the design process and reducing the computational complexity. This possibility is the result of the skew symmetric property of the fuzzy rule tables of the system.
\end{abstract}

Keywords: Inverted Pendulum System, Segway Mobile Robot, Fuzzy Logic Control System, Position Control, Balance Control

\section{Introduction}

Studies on the control of inverted pendulum type systems have been widely reported. This is because this type of system is a good model to verify the performance of a proposed controller for such a system, which is inherently a nonlinear. An inverted pendulum type mobile robot system adds mobility to the utilization of a mechanical function that balances the inverted pendulum system[1]. Furthermore, it is similar to the control scheme of a biped robot which is modelled after the human form and is supported by two feet.

Received: Apr. 23, 2015

Revised : Jun. 24, 2015

Accepted: Jun. 25, 2015

Correspondence to: Byung-Jae Choi (bjchoi@daegu.ac.kr)

(CThe Korean Institute of Intelligent Systems

CCThis is an Open Access article distributed under the terms of the Creative Commons Attribution Non-Commercial License (http://creativecommons.org/licenses/ by-nc/3.0/) which permits unrestricted noncommercial use, distribution, and reproduction in any medium, provided the original work is properly cited.

Segway type mobile robots operate based on the dynamics of the inverted pendulum system. They are capable of forward, backward, and turning motions, and these are the only possible movements of the body. Unlike scooters whose two interdependent wheels are in series, Segway mobile robots have two wheels connected in a parallel configuration. Thus, Segway mobile robots allow the construction of a mobile platform that can travel smoothly to a small area by reducing the migration area. However, a mobile robot employing an inverted pendulum mechanism as its mobile platform requires an additional controller design to maintain the balance of the body, as its balancing ability is generally not good under excessive disturbances.

In this paper, we propose the design of a fuzzy logic control system for the position and balance control of an inverted pendulum type Segway mobile robot. We first introduce a dynamic model of the Segway mobile robot and analyze it. We then design two fuzzy logic control systems based on our analysis. Their usefulness is verified by simulation examples. 


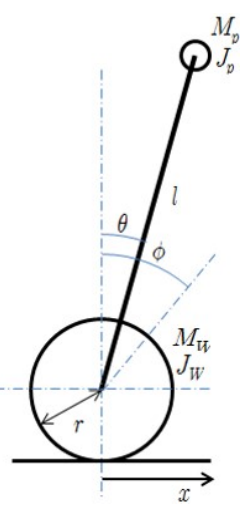

(a)

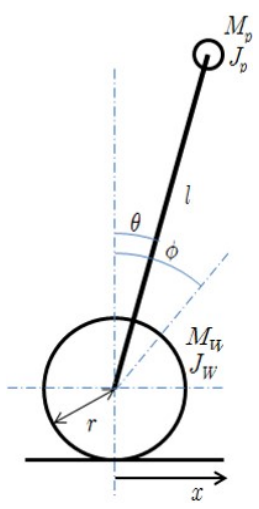

(b)

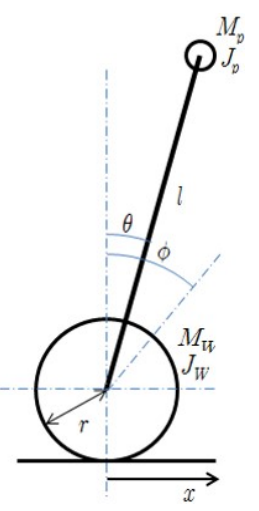

(c)
Figure 1. Schematics of Segway type mobile robot.

Based on the skew symmetry property of the rule table for the fuzzy logic control system, we also present a possibility for a reduction in the computational complexity and the simplification of the design of the fuzzy logic control system.

The remainder of the paper is organized as follows. In Section 2, we describe the introduction of the dynamic model of the Segway mobile robot. The design of two fuzzy logic control systems for the Segway mobile robot is presented in Section 3. In Section 4, we present the results of simulation examples and explain their relevance. Concluding remarks are given in Section 5.

\section{Dynamics of Segway Mobile Robot}

Segway type mobile robots are composed of two wheels and a pole between them. The angle of the pole is measured by a gyro, tilt or acceleration sensor, and is maintained at zero degrees.

In this section, we introduce the dynamics of the Segway type mobile robot which is an inverted pendulum type mobile robot. Schematics of this robot are shown in Figure 1.

The main parameters used in Figure 1 are presented in Table 1 .

As shown in Figure 1, the mobile robot can be divided into two parts. The wheel part and the pole part, which consists of a pole and a driving motor to support the body over the wheel and maintain the balance of the robot.

We first introduce equations associated with the wheel part of the robot, which is shown in Figure 1 (b). The following equations of motion are derived from the moment of inertia of the wheel of the driving shaft and the reaction forces of the
Table 1. Definitions of Segway robot Parameters

\begin{tabular}{cl}
\hline Parameter & Definition \\
\hline$x$ & Displacement of robot \\
$M_{w}$ & Mass of wheel \\
$J_{w}$ & Moment of inertia of wheel \\
$r$ & Radius of wheel \\
\hline & Length of pole \\
$M_{p}$ & Mass of center of gravity of pole \\
$J_{p}$ & Moment of inertia of center of gravity \\
& of pole \\
\hline$g$ & Acceleration due to gravity \\
\hline$\phi$ & Angle of pole \\
\hline$V$ & Rotational angle of wheel \\
$H$ & Vertical reaction force of pole \\
\hline$H_{w}$ & Horizontal reaction force of pole \\
$f_{w}$ & Horizontal reaction force of wheel \\
$f_{p}$ & Unknown external force of wheel \\
$\tau$ & Torque of wheel \\
\hline$x_{p}$ & $x$-axis displacement of pole \\
$y_{p}$ & $y$-axis displacement of pole \\
\hline
\end{tabular}

horizontal and vertical axes of the pole:

$$
\begin{gathered}
M_{w} \ddot{x}=H+f_{w}+H_{w} \\
J_{w} \ddot{\phi}=-r H_{w}+\tau .
\end{gathered}
$$

The rotational angle of the wheel and the displacement of the robot have the following relationship.

$$
r \ddot{\phi}=\ddot{x} \text {. }
$$

From Eqs. (2) and (3), the horizontal reaction force of the wheels is derived from the displacement and the driving torque as

$$
H_{w}=\frac{\tau}{r}-\frac{J_{w}}{r^{2}} \ddot{x}
$$

The following equation can be obtained from Eqs. (1) and (4).

$$
\left(M_{w}+\frac{J_{w}}{r^{2}}\right) \ddot{x}=H+f_{w}+\frac{\tau}{r} .
$$

We then derive the dynamic equations of the pole. The force acting the pole is shown in Figure 1 (c). Then the horizontal displacement of the center of gravity of the pole is

$$
x_{p}=x+l \sin \theta \text {. }
$$


Eq. (6) can be expressed in an acceleration form as

$$
\ddot{x}_{p}=\ddot{x}+l \ddot{\theta} \cos \theta-l \dot{\theta}^{2} \sin \theta .
$$

Eq. (7) can be expressed as an equation of motion with respect to the axis of the center of gravity of the pole as

$$
\begin{gathered}
M_{p} \ddot{x}_{p}=f_{p}-H \\
H=-\left(M_{p} \ddot{x}+M_{p} l\left(\ddot{\theta} \cos \theta-\dot{\theta}^{2} \sin \theta\right)\right)+f_{p} .
\end{gathered}
$$

Next, the vertical displacement of the center of gravity of the pole is

$$
y_{p}=l \cos \theta .
$$

Eq. (10) can be expressed in the form of an acceleration equation as

$$
\ddot{y}_{p}=-l\left(\dot{\theta}^{2} \cos \theta+\ddot{\theta} \sin \theta\right) .
$$

Eq. (11) can be expressed as an equation of motion with respect to the $y$ axis of the center of gravity of the pole as

$$
\begin{gathered}
M_{p} \ddot{y}_{p}=V-M_{p} g . \\
V=-M_{p} l\left(\dot{\theta}^{2} \cos \theta+\ddot{\theta} \sin \theta\right)+M_{p} g
\end{gathered}
$$

The following equation is derived from Eqs. (5) and (9).

$$
\begin{aligned}
\left(M_{w}+M_{p}+\frac{J_{w}}{r^{2}}\right) \ddot{x} & +M_{p} l \cos \theta \ddot{\theta}-M_{p} l \sin \theta \dot{\theta}^{2} \\
& =f_{w}+f_{p}+\frac{1}{r} \tau
\end{aligned}
$$

The moment of inertia of the center of gravity of the pole is

$$
J_{p} \ddot{\theta}=V l \sin \theta-H l \cos \theta-\tau \text {. }
$$

The following equation is derived from Eqs. (9), (13) and (15).

$$
\left(J_{p}+M_{p} l^{2}\right) \ddot{\theta}+M_{p} l \ddot{x} \cos \theta-M_{p} g l \sin \theta=l \cos \theta f_{p}-\tau
$$

The dynamic model of the Segway robot is expressed by Eqs. (14) and (16).

\section{Design of Fuzzy Logic Control Systems}

Two fuzzy logic control (FLC) systems are required to control the Segway robot: the Distance FLC and the Balance FLC for position and balance control, respectively.

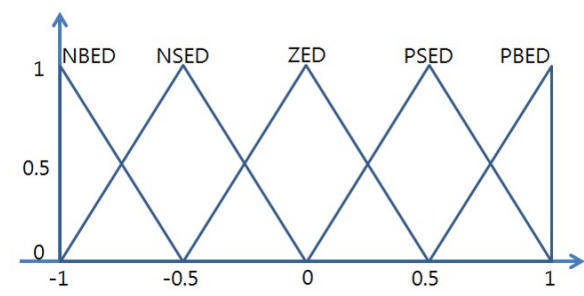

Figure 2. Membership functions of input variable, edist, for Distance FLC.

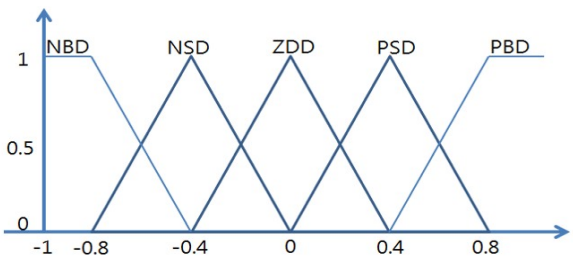

Figure 3. Membership functions of input variable, dedist, for Distance FLC.

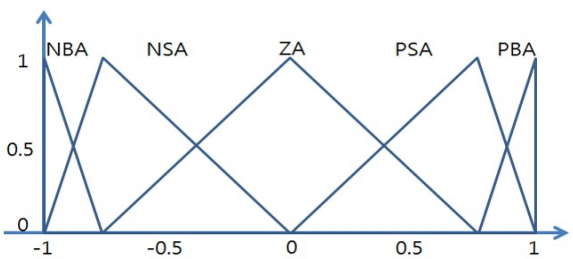

Figure 4. Membership functions of output variable, AddAngle, for Distance FLC.

The Distance FLC to control the position of the robot is designed first. It has two input variables, edist and dedist, which are the error signal between the set position and the current position of the robot and its change signal, respectively. It also has one output variable, AddAngle, which is the weight of the angle error of the pole.

The membership functions of the input and output variables of the Distance FLC are shown in Figures 2, 3, and 4.

The control rule table for the Distance FLC is given in Table 2 .

We then design the Balance FLC for the control of the balance of the robot. Its input variables are etheta and detheta, which represent the angle of the pole and the output of the Distance FLC. Its output variable is the torque.

The membership functions of the input and output variables of the Balance FLC are shown in Figures 5, 6, and 7. 
Table 2. Rule table for Distance FLC

\begin{tabular}{|c|c|c|c|c|c|}
\hline edist $\quad$ dedist & NBD & NSD & ZDD & PSD & PBD \\
\hline NBED & PBA & PBA & PBA & PSA & ZA \\
\hline NSED & PBA & PBA & PSA & $\mathrm{ZA}$ & NSA \\
\hline ZED & PBA & PSA & $\mathrm{ZA}$ & NSA & NBA \\
\hline PSED & PSA & ZA & NSA & NBA & NBA \\
\hline PBED & $\mathrm{ZA}$ & NSA & NBA & NBA & NBA \\
\hline
\end{tabular}

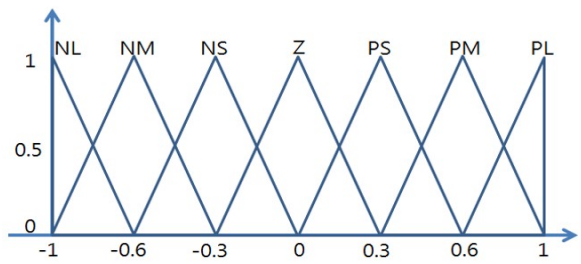

Figure 5. Membership functions of input variable, etheta, for Balance FLC.

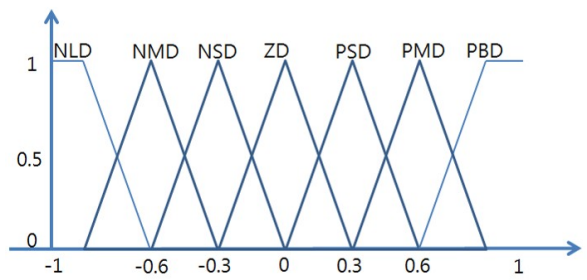

Figure 6. Membership functions of input variable, detheta, for Balance FLC.

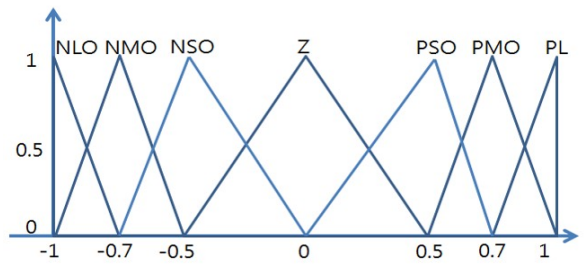

Figure 7. Membership functions of output variable, torque, for Balance FLC.

The $\mathrm{Z}$ membership function for the output variable, torque, was widely set. The shapes of the membership functions of PLO and NLO are sharper than that of Z membership function. This can reduce the vibration of the output variable and make the output of the system converge to a steady state.

The control rule table for the Balance FLC is given in Table 3.
Table 3. IRule table for Balance FLC

\begin{tabular}{|c|c|c|c|c|c|c|c|}
\hline etheta detheta & NLD & NMI & NSD & ZD & PSD & PMD & PLD \\
\hline NL & NLO & NLO & NMO & NMO & NSO & NSO & $\mathrm{Z}$ \\
\hline NM & NLO & NMC & NMC & NSO & NSO & $\mathrm{Z}$ & PSO \\
\hline NS & NMO & NMO & NSO & NSO & $\mathrm{Z}$ & PSO & PSO \\
\hline $\mathbf{Z}$ & NMC & NSO & NSO & $\mathrm{Z}$ & PSO & PSO & PMC \\
\hline PS & NSO & NSO & $\mathrm{Z}$ & PSO & PSO & PMO & PMO \\
\hline PM & NSO & $\mathrm{Z}$ & PSO & PSO & PMO & PMO & PLO \\
\hline PL & Z & PSO & PSO & PMO & PMO & PLO & PLO \\
\hline
\end{tabular}

\section{Simulation Examples}

In this section, we simulate the position and balance control of the Segway mobile robot using the two proposed fuzzy logic control systems. We first set $\theta, \dot{\theta}$, and $\tau$ as

$$
-1 \leq \theta \leq 1, \quad-3 \leq \theta \leq 3, \quad-0.5 \leq \theta \leq 0.5
$$

And some parameters of the Segway mobile robot are as follows[2]:

$$
\begin{aligned}
M_{w} & =0.076 \mathrm{~kg}, J_{w}=3.42 \times 10^{-5} \mathrm{~kg} \cdot \mathrm{m}^{2}, \\
r & =0.03 \mathrm{~m}, l=0.15 \mathrm{~m}, M_{p}=0.6 \mathrm{~kg}, \\
J_{p} & =1.34 \times 10^{-2} \mathrm{~kg} \cdot \mathrm{m}^{2}, g=9.81 \mathrm{~m} / \mathrm{s}^{2} .
\end{aligned}
$$

The AND and OR operations for the fuzzy logic control system are min and max, respectively. Additionally, Mamdani reasoning and the defuzzification method for the center of gravity are used in this simulation.

The results of the weight of the angle error of the pole, the torque, the displacement, the change in the displacement, the angle of the pole, and the change in this angle are shown in Figures $8,9,10,11,12$, and 13 respectively. The initial conditions are as follows: the angle of the pole $=1$ [radian], the target position is $10[\mathrm{~m}]$.

\section{Concluding Remarks}

In this paper, we analyzed the dynamics of the Segway mobile robot which has nonlinear properties, and designed two fuzzy logic control systems for the control of the position of the Segway mobile robot and the balance of the pole part of the robot. First, we designed the Distance FLC for the position control. The two input variables of the Distance FLC are the error signal between the set position and the current position of the robot and its change signal, the output variable is the 


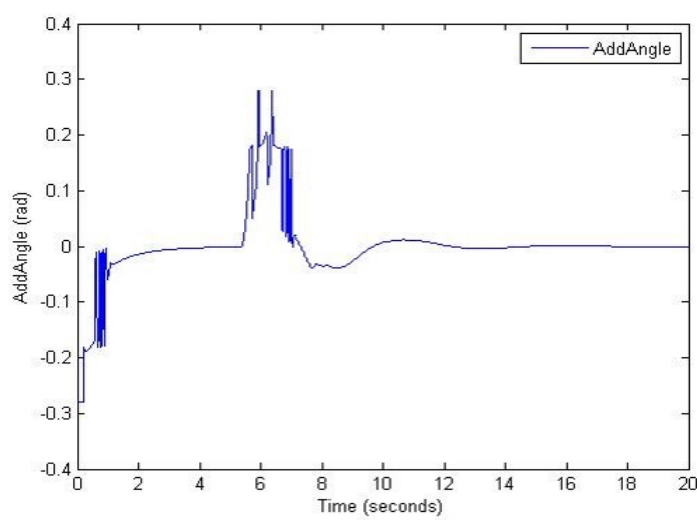

Figure 8. Simulation results of AddAngle.

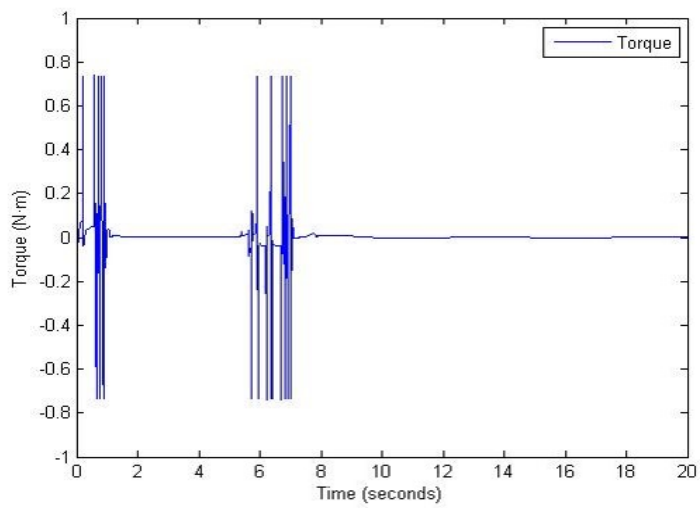

Figure 9. Simulation results of torque.

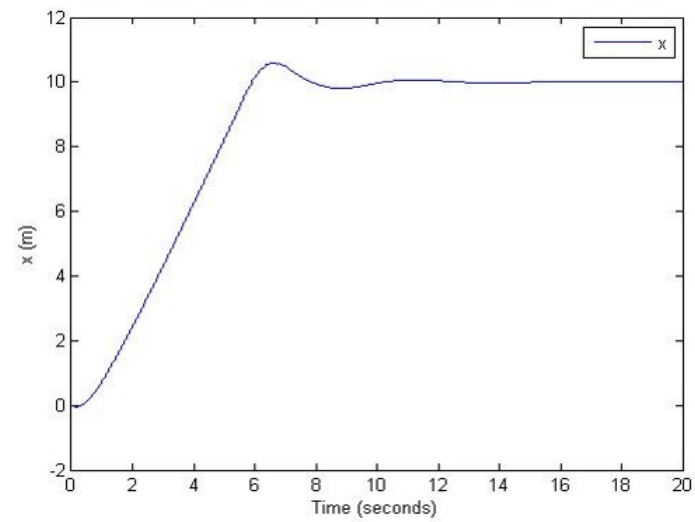

Figure 10. Simulation results of displacement of robot.

weight of the angle error of the pole. We then designed the Balance FLC for the control of the balance of the pole. Its input variables of the Balance FLC are the angle of the pole and

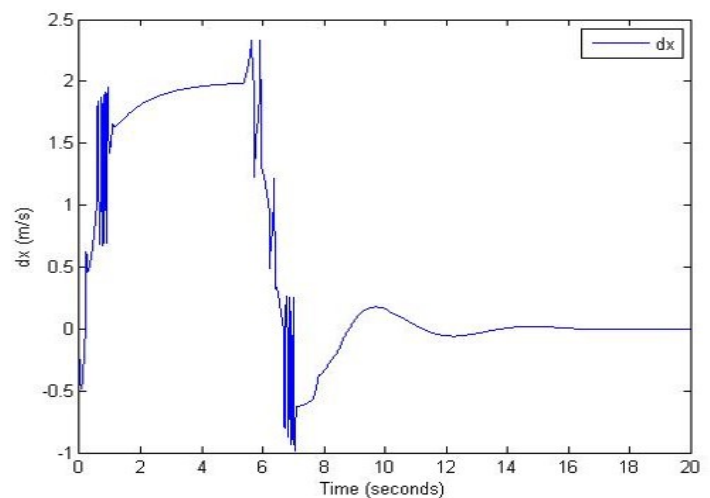

Figure 11. Simulation results of velocity of robot.

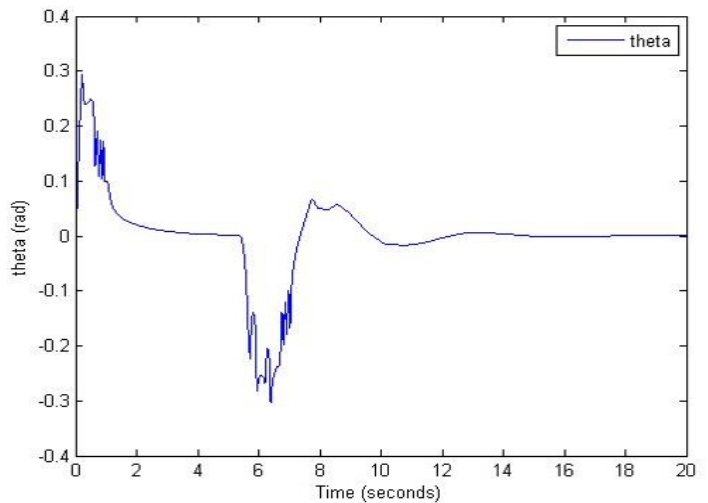

Figure 12. Simulation results of angle of pole.

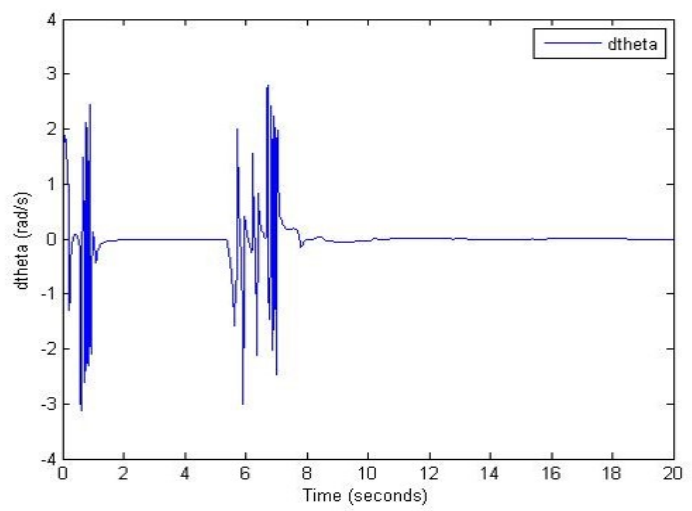

Figure 13. Simulation results of angular velocity of pole.

the output of the Distance FLC, and the output variable is the torque. The control rule table indicates that the control rules for the two fuzzy logic control systems are the skew symmetric[3]. 
This can simplify the control rule table to a single dimensional case with a small number of input variables and control rules. This in turn simplifies the design of the overall control system. This will be studied in future work with the implementation of an embedded board based system.

\section{Acknowledgement}

This research was supported by the Daegu University Research Scholarship Grants.

\section{References}

[1] The SEGWAY website. [Online]. Available: http://www. segway.com/

[2] S.-H. Lee and S.-Y. Rhee, "Dynamic modelling of a wheeled inverted pendulum for inclined road and changing its center of gravity," J. of Korean Institute of Intelligent Systems, vol. 22, no. 1, pp. 69-74, 2012.

[3] B.-J. Choi and S. Jin, "Design of Simple-structured Fuzzy Logic System based Driving Controller for Mobile Robot," J. of Korean Institute of Intelligent Systems, vol.22, no.1, 2012.2.

[4] H. W. Kim and S. Jung, "Fuzzy Logic Application to a Two-wheel Mobile Robot for Balancing Control Performance," Int. J. of Fuzzy Logic and Intelligent Systems, vol.12, no.2, 2012.6.

[5] J. H. Park, "Fuzzy-logic zero-moment-point trajectory generation for reduced trunk motion of biped robots," Fuzzy Set Techniques for Intelligent Robotic Systems, vol. 134, no. 1, pp. 189-203, Feb. 2003.

[6] H. Ha and J. Lee, "A control of mobile inverted pendulum using single accelerometer," J. of Institute of Control, Robotics and Systems, vol. 16, no. 5, 2010

[7] S. W. Nawawi, M. N. Ahmad, and J. H. S. Osman, "Control of two-wheels inverted pendulum mobile robot using full order sliding mode control," Proc. of International Conference on Man-Machine System, Lankawi, Malaysia, Sep. 2006.

[8] P. Axelsson and Y. Jung, Lego Segway Project Report, Technical Report from Automatic Control at Linkopings Universitet, Division of Automatic Control, 2011.

[9] J. S. Noh, G. H. Lee, and S. Jung, "Position control of a mobile inverted pendulum system using radial basis func-

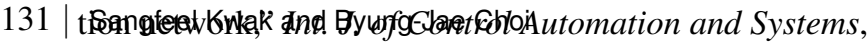
vol. 8 , no. $1,2010$.
[10] J. Huang, Z. Guan, T. Matsuno, T. Fukuda, and K. Sekiyama, "Sliding-Mode Velocity Control of MobileWheeled Inverted-Pendulum Systems," IEEE Trans. on Robotics, vol. 26, no. 4, 2010.

[11] T. Jin, "Obstacle Avoidance of Mobile Robot Based on Behavior Hierarchy by Fuzzy Logic," Int. J. of Fuzzy Logic and Intelligent Systems, vol.12, no.3, 2012.9

[12] L. Mao, J. Huang, F. Ding, and Y. Wang, "Velocity control of mobile wheeled inverted pendulum," Int. J. of Modelling Identification and Control, vol. 19, no. 1, 2013.

[13] X. Xiong and B.-J. Choi, "Comparative Analysis of Detection Algorithms for Corner and Blob Features in Image Processing," Int. J. of Fuzzy Logic and Intelligent Systems, vol.13, no.4, 2013.

[14] B.-H. Kim, "Analysis of Balance of Quadrupedal Robotic Walk using Measure of Balance Margin,' Int. J. of Fuzzy Logic and Intelligent Systems, vol.13, no.2, 2013.

[15] K. D. Do, and G. Seet, "Motion Control of a Two-Wheeled Mobile Vehicle with an Inverted Pendulum," J. of Intelligent and Robotic Systems, vol. 60, no. 3-4, 2010.

[16] H.-G. Nguyen, W.-H. Kim, and J.-H. Shin, "A Study on an Adaptive Robust Fuzzy Controller with GAs for Path Tracking of a Wheeled Mobile Robot," Int. J. of Fuzzy Logic and Intelligent Systems, vol.10, no.1, 2010.3.

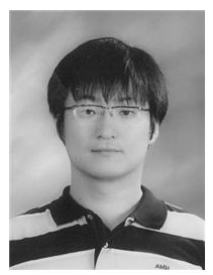

Sangfeel Kwak is a Ph.D candidate at the College of Information, Communication, and Computer Engineering, Daegu University. He received the M.S. degree in Electronics Engineering from Daegu University, Korea. Currently, his main research interests include intelligent control and systems.

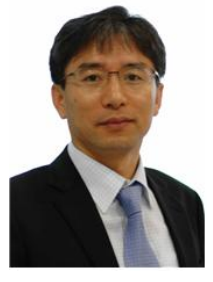

Byung-Jae Choi is a professor at the College of Information, Communication, and Computer Engineering, Daegu University. He received the Ph.D. degree in Electrical Engineering from KAIST, Korea. Currently, his main research interests include intelligent control and systems. 